\title{
Mercer's Theorem and Fredholm resolvents
}

\section{C.S. Withers}

\begin{abstract}
Multivariate versions of Mercer's Theorem and the usual expansions of the resolvent and Fredholm determinant are shown to hold for an $n \times n$ symmetric kernel $N(x, y)$ with arbitrary domain in $R^{p}$ under weakened continuity conditions. Further, the resolvent and determinant of $N(x, y)-a(x) b(y)$ are given in terms of those of $N(x, y)$.
\end{abstract}

\section{Introduction}

Our main result, given in $\$ 3$, deals with eigenfunction expansions of the $n \times n$ matrix kernel $N(x, y)$ (Mercer's Theorem) and its iterates $N_{j}(x, y)$ and resolvent $N(x, y, \lambda)$. As well as allowing $n$ to be arbitrary and the domain to be unbounded, we weaken the usual continuity assumptions of this important theorem, which has found applications in optimum detection theory (for example, Deutsch [2], p. 244) and statistics. These expansion formula are basic to the study of the distribution of the random variable $\int|X(t)|^{2} d t$ where $X: R^{p} \rightarrow R^{n}$ is a Gaussian process with covariance $N$. Such random variables arise in connection with the asymptotic distribution and power of certain statistical tests; see withers [8].

In $\$ 4$ we give a simple but useful result: formulae for the resolvent and Fredholm determinant of $N(x, y)-a(x) b(y)$ in terms of those of $N(x, y)$, where $a(x), b(y)$ are $n \times q$ and $q \times n$ functions. This 
section was motivated by the study of the random variable $\int|Y(t)|^{2} d t$ where

$$
Y(t)=X(t)+f(t) \int g(x) X(s) d s,
$$

and $f, g$ are matrix functions, and $X$ is as above; see Withers [9] for statistical applications where such variables arise naturally. The basic results of Fredholm integral equation theory for a matrix kernel $N(x, y)$ defined on a domain $\Omega \times \Omega$ where $\Omega$ is an arbitrary domain in $p$-dimensional euclidean space $R^{p}$ appear to have been stated only for the case $n=p=1$ and $\Omega$ a bounded interval. However the technique of deducing these results for general $n$ is well known and some authors have realised such extensions are possible (cf. Riesz and Nagy [6], p. 145), although others have suggested that $\Omega$ must be bounded ( $c f$. Pogorzelski [5], p. 95). We therefore begin with a summary of these results for general $n$ and arbitrary domain in $p$.

\section{Some basic results}

We give here generalisations of some basic results. These are easily deduced by the method of Carleman [1] (given for $n=p=1, \Omega=[a, b]$, $N$ real), the technique of reducing to $n=1$ (for example, Pogorzelski [5], p. 181) and the standard proofs.

Throughout this paper we shall use $A^{*}$ to denote the conjugate transpose of a complex matrix $A=\left(A_{i j}\right)$, and $\|\cdot\|$ to denote the norm defined by $\|A\|^{2}=\sum\left|A_{i j}\right|^{2}$. All integrals will be with respect to Lebesgue measure over $\Omega$, an arbitrary subset of $R^{p}$.

Given $\Omega \subset R^{P}$ consider a complex measurable $n \times n$ function $N(x, y)$ on $\Omega \times \Omega$ such that

$$
0<\iint\|N(x, y)\|^{2} d x d y<\infty .
$$

For $f$ a complex measurable $n \times q$ function on $\Omega$ such that $\int\|f\|^{2}<\infty$, let $N f(x)=\int N(x, s) f(s) d s$ and $f(y) * N=\int f(s) * N(s, y) d s$. 
Let $N_{j}=N^{j-1} N, j \geq 1$, where $N^{0}=I$, the identity operator. Then $N(x, y, \lambda)$, the resolvent of $N(x, y)$ exists and for $y$ in $\Omega$, $h(x)=N(x, y, \lambda)$ is the unique solution of

$$
h=N(\cdot, y)+\lambda N h \text {, }
$$

and for $x$ in $\Omega, g(y)=N(x, y, \lambda)$ is the unique solution of

$$
g=N(x, \cdot)+\lambda g N \text {. }
$$

When

$$
\sum \int\left|N_{i i}(x, x)\right| d x<\infty
$$

then the Fredholm determinant $D(\lambda)$ exists and is given by

$$
\frac{d}{d \lambda} \log D(\lambda)=-\int \operatorname{trace} N(x, x, \lambda) d x, D(0)=1 .
$$

When

$$
N^{*}(y, x)=N(x, y) \text { for } x, y \text { in } \Omega \text {, }
$$

then there exist real numbers $\left\{\lambda_{1}, \lambda_{2}, \ldots\right\}$ (eigenvalues) and complex $n$-vectors on $\Omega,\left\{\phi_{1}, \phi_{2}, \ldots\right\}$ (eigenvectors) satisfying

$$
\lambda_{i} N \phi_{i}=\phi_{i}, \int \phi_{i}^{*} \phi_{j}=\left\{\begin{array}{ll}
1, & i=j \\
0, & i \neq j
\end{array}\right\}, \quad 0<\left|\lambda_{1}\right| \leq\left|\lambda_{2}\right| \leq \ldots ;
$$

such that if

$$
\lambda N \phi=\phi, \int|\phi|^{2}<\infty, \phi n \times 1,
$$

then $\lambda=\lambda_{k}$ for some $k$, and $\phi$ is a linear combination of those $\phi_{r}$ such that $\lambda_{r}=\lambda$.

When (3) and

$$
\sup _{y} \int\|N(x, y)\|^{2} d x<\infty,
$$

then for $x$ in $\Omega$, and almost all $y$ in $\Omega$, 


$$
N(x, y, \lambda)=N(x, y)+\lambda \sum_{1}^{\infty}\left\{\frac{\phi_{i}(x) \phi_{i}(y)^{*}}{\lambda_{i}\left(\lambda_{i}-\lambda\right)}\right\}, \lambda \text { not an eigenvalue, }
$$

and

$$
N_{j}(x, y)=\sum_{1}^{\infty} \lambda_{i}^{-j} \phi_{i}(x) \phi_{i}(y) *, j \geq 2,
$$

and the convergence of these series is (element-wise) absolute and uniform in $\Omega^{2}$

\section{Mercer's Theorem}

Mercer's Theorem concerns the expansion of $N$ in terms of its eigenfunctions and eigenvalues:

$$
N(x, y)=\sum \phi_{i}(x) \phi_{i}(y) * / \lambda_{i}
$$

Statements of the theorem in the literature all make unnecessary continuity and other assumptions. For example sometimes (5) is assumed (for example, Pogorzelski [5], p. 150). Our aim here is to impose as few conditions on $N$ as seems possible. It is worth noting that a useful weakening of our continuity assumptions (9)-(11) may be made by excluding from $\Omega$ the set of points $P$ at which they do not hold, provided $P$ has Lebesgue measure zero.

Our version of Mercer's Theorem is as follows.

THEOREM 1. Suppose $N$ satisfies (1), (3), and the following

$$
\int \phi^{*} \phi=\iint \phi^{*}(x) N(x, y) \phi(y) d x d y \geq 0
$$

for all complex $n \times 1$ functions $\phi$ such that $\int|\phi|^{2}<\infty$,

$$
\sup \underset{x \in \Omega}{\operatorname{trace}} N(x, x)<\infty \text {, }
$$

$$
N(x, y) \text { is continuous at } y=x \in \Omega,
$$

$$
N_{2}(x, x)_{i i} \text { is continuous in } \Omega, 1 \leq i \leq n \text {. }
$$

$$
N_{2}(x, y)_{i i} \text { is continuous at } y=x \text { in } \Omega, \quad 1 \leq i \leq n \text {, }
$$


then for $\left\{\lambda_{i}, \phi_{i}\right\}$ above, $\left\{\phi_{i}\right\}$ are continuous and $\sum_{1}^{\infty} \lambda_{i}^{-1} \phi_{i}(x) \phi_{i}(y)$ * converges (elementwise) absolutely and ziformly in $\Omega^{2}$ and equals $N(x, y)$ almost everywhere in $\Omega^{2}$.

NOTES. (i) Since a uniformly convergent series of continuous functions converges to a continuous function, equality holds at continuity points of $N$, such as $x=y$.

(ii) Since (4) implies $\phi=\lambda^{m} N_{\phi}^{m}$, (10) and (11) may be replaced by $(11)^{1}$ for some $m \geq 1, N_{2 m}(x, y)_{i i}$ and $N_{2 m}(x, x)_{i i}$ are continuous at $y=x$ for $x$ in $\Omega, I \leq i \leq n$; (cf. Hobson [3] who gives for continuity of $\left.\left\{\phi_{i}\right\}\right)$;

$(11)^{2}$ for some $m \geq 1, N_{m}(x, y)$ is continuous in $x$ for almost all $y$. in $\Omega$ and for $j \geq m, N_{j}$ is bounded.

Proof of Theorem 1. We may without loss take $n=1 \cdot\left\{\phi_{i}\right\}$ are continuous because (4) implies

$$
|\phi(x)-\phi(y)|^{2} \leq|\lambda|^{2} 1(x, y) \int|\phi|^{2},
$$

where $I(x, y)=\int|N(x, s)-N(y, s)|^{2} d s \rightarrow 0$ by (3), (10), (11).

By (9) and the standard method (for example, [5], p. 151), $N(x, x)$ and $N(x, x)-\sum_{1}^{q} \lambda_{i}^{-1}\left|\phi_{i}(x)\right|^{2}$ are real and non-negative for $q \geq 1$. Hence by (8) for $\varepsilon>0$, there exists $M$ such that

$$
\sum_{M}^{\infty} \lambda_{i}^{-1}\left|\phi_{i}(x)\right|^{2}<\varepsilon \text { in } \Omega
$$

so that for $n_{2} \geq n_{1} \geq M$,

$$
\sum_{n_{1}}^{n_{2}} \lambda_{i}^{-1}\left|\phi_{i}(x) \phi_{i}(y)^{*}\right|<\varepsilon \text { in } \Omega^{2}
$$


Hence $\sum \lambda_{i}^{-1} \phi_{i}(x) \phi_{i}(y)^{*}$ converges absolutely and uniformly in $\Omega^{2}$, so that by [5], pp. 130,131 , the sum equals $N(x, y)$ almost everywhere.

The usual expansions now follow:

COROLLARY. Under the conditions of Theorem 1 ,

(12) for $j \geq 1, N_{j}(x ; y)=\sum \lambda_{i}^{-j} \phi_{i}(x) \phi_{i}(y)^{*}$ almost everywhere in $\Omega^{2}$,

(13) $\quad N(x, y, \lambda)=\left[\left(\lambda_{i}-\lambda\right)^{-1} \phi_{i}(x) \phi_{i}(y)^{*}\right.$ almost everywhere in $\Omega^{2}$, if $\lambda$ is not an eigenvalue,

(14) for $j \geq 1, \int$ trace $N_{j}(x, x) d x=\sum \lambda_{i}^{-j}$, (possibly infinite for $j=1$ ), and the (elementwise) convergence in (12) and (13) is absolute and uniform.

If also (2) holds, that is, $\int \operatorname{trace} N(x, x) d x<\infty$, then

$$
D(\lambda)=\prod_{1}^{\infty}\left(1-\lambda / \lambda_{i}\right) .
$$

\section{The kernel $N(x, y)-a(x) b(y)$}

Carleman [1] gave expansions in $\lambda$ for $D(x, y, \lambda)$ and $D(\lambda)$ for $N=G+H$ and $N=G \cdot H$ in terms of multiple integrals of determinants, akin to Fredholm's series. Here we give more convenient formulae for $K(x, y, \lambda)$ and $D_{K}(\lambda)$, the resolvent and Fredholm determinant for the particular case

$$
K(x, y)=N(x, y)-a(x) b(y),
$$

where we assume $a, b$ are $n \times q$ and $q \times n$ functions on $\Omega$ such that

$$
\int\|a\|^{2}<\infty, \int\|b\|^{2}<\infty \text {, }
$$

when $D_{N}(\lambda), N(x, y, \lambda)$ are known, and where we set $D_{N}(\lambda)=D(\lambda)$ to avoid confusion with $D_{K}(\lambda)$.

THEOREM 2. Let $N$ satisfy (1) and (2). Let $T$ denote the operator 
$(I-\lambda N)^{-1}$ so that

$$
T a(x)=a(x)+\lambda \int N(x, y, \lambda) a(y) d y
$$

and

$$
\begin{gathered}
b(x) T=b(x)+\lambda \int b(y) N(y, x, \lambda) d y . \\
\text { Let } B(\lambda)=1_{q}+\lambda \int b T_{a} \text {, where } 1_{q}=\operatorname{diag}(1, \ldots, 1) \text {. Then }
\end{gathered}
$$

$$
K(x, y, \lambda)=N(x, y, \lambda)-T a(x) B(\lambda)^{-1} b(y) T,
$$

for $x, y$ in $\Omega$ and $D_{K}(\lambda) \neq 0$. Also

$$
D_{K}(\lambda)=D_{N}(\lambda) \cdot \operatorname{det} B(\lambda)
$$

Further, if $\operatorname{det} B(\lambda)=0$, then eigenfunctions of $K$ with eigenvalue $\lambda$ alz have the form Tac where $c \neq 0$ is a q-vector such that

$$
B(\lambda) c=0 \text {. }
$$

NOTE. Michlin [4] has given a special case of (16) without proof.

Proof. Suppose $D_{N}(\lambda) \neq 0$ and $D_{K}(\lambda)=0$.

Then $f=\lambda K f$ has a non-trivial solution where $K f(x)=\int K(x, y) f(y) d y$. Hence $c=\int b f \neq 0$ and $f=-\lambda T a c$. Hence $\operatorname{det} B(\lambda)=0$.

Suppose $D_{N}(\lambda) \cdot \operatorname{det} B(\lambda) \neq 0$. Then $D_{K}(\lambda) \neq 0$ and for $h$ such that $\int|h|^{2}<\infty, f=h+\lambda K f$ has solution

$$
f=(I-\lambda K)^{-1} h=T(h-\lambda a c)
$$

Hence $c=R h$ where $R=B(\lambda)^{-1} b T$, so that $(I-\lambda K)^{-1}=T(I-\lambda a R)$, which proves (16).

$$
\text { If } \begin{aligned}
& D_{K}(\lambda) D_{N}(\lambda) \neq 0, \\
& \begin{aligned}
\frac{d}{d \lambda} \log \left(D_{K}(\lambda) / D_{N}(\lambda)\right) & =-\int \operatorname{trace}(K(x, x, \lambda)-N(x, x, \lambda)) d x \\
& =\operatorname{trace} B(\lambda)^{-1} C, \text { by }(14),
\end{aligned}
\end{aligned}
$$


where $c=\int b T^{2} a$. For $|\lambda|$ small,

$$
\frac{d}{d \lambda} \lambda T=I+2 \lambda N+3 \lambda^{2} N^{2}+\ldots=T^{2},
$$

so that $C=d / d \lambda B(\lambda)$ for all $\lambda$ by analytic continuation. (17) follows.

\section{References}

[1] T. Carleman, "Zur Theorie der linearen Integralgleichungen", Math. Z. 9 (1921), 196-217.

[2] Ralph Deutsch, Estimation theory (Prentice-Hall, Englewood Cliffs, New Jersey, 1965).

[3] E.W. Hobson, "On the representation of the symmetrical nucleus of a linear integral equation", Proc. London Math. Soc. (2) 14 (1915), $5-30$.

[4] S. Michlin, "On the convergence of Fredholm series", C.R. (Doklady) Acad. Sci. URSS (NS) 42 (1944), 373-376.

[5] W. Pogorzelski, Integral equations and their applications. Vol. I (International Series of Monographs in Pure and Applied Mathematics, 88. Pergamon, Oxford, New York, Frankfurt; PWNPolish Scientific Publishers, Warsaw, 1966).

[6] Frigyes Riesz and Béla Sz.-Nagy, Functional analysis (Frederick Ungar, New York, 1955).

[1] F. Smithies, Integral equations (Cambridge Tracts in Mathematics and Mathematical Physics, 49. Cambridge University Press, Cambridge, 1958).

[8] C.S. Withers, "The characteristic function of the $L_{2}$-norm of a Gaussian process", submitted.

[9] C.S. Withers, "On the asymptotic power of statistics which are $L_{2}$-norms", submitted.

Applied Mathematics Division, Department of Scientific and Industrial Research, Wellington, New Zealand. 